\title{
Nene Rille und neuer Krater, entdeckt auf den Mondphotographien der Lick Sternwarte.
}

\author{
Von L. Weinek.
}

Herr Professor Edward S. Holden hatte im November 1889 die Güte, mir das freundliche Anerbieten zu machen, eine Reihe der besten, mit dem 36 Zöller aufgenommenen Mond-Negative nach Prag senden zu wollen, auf dass dieselben hier ebensowohl als Grundlage für meine Zeichnungen am Fernrohr wie auch zu selbstständigen Specialstudien der Mondoberfiäche verwendet würden. Seit Beginn des Jahres 1890 sind bereits 32 Mondplatten der LickSternwarte, welche fast jeden Tag der. Lunation illustriren, in meine Hände gelangt.

Nachdem ich für den gedachten Zweck einen geeigneten Apparat gebaut, konnten am I 2. Juni 1890 die bezüglichen Arbeiten in Angriff genommen werden. Zuerst wurde das Mare Crisium nach der Lick-Aufnahme vom 23. August 1888 (Mondalter $=16$ Tage) in vierfacher Vergrösserung während einer Arbeitsdauer von $34 \%$ Stunden ausgeführt. Hierauf wurde die Wallebene Archimedes zweimal und mit entgegengesetztem Schattenwurf, ebenso Arzachel nach den Lickaufnahmen vom 15. und 27. August 1888 (Mondalter $=8$, bezw. 20 Tage) in zehnfacher Vergrösserung gezeichnet. Letztere vier Bilder von je 5 zu 7 Centimeter Grösse beanspruchten zusammen $179 \%$ Stunden. Die erwähnten Zeichnungen oder richtiger Tuschirungen sind bereits beim k. u. k. militär-geographischen Institute in Wien zur heliographischen Reproduction und sollen in den Analen der Hamiltoner und der Prager Sternwarte publicirt werden. Die Fortsetzung der angeführten Detailabbildungen geschieht gegenwärtig nach beträchtlich gesteigertem Maass. stabe, nachdem ein entsprechendes ausgezeichnetes Ocular von der optischen Anstalt Reinfelder \& Hertel in München beschaft werden konnte.

Im Laufe dieser Arbeiten wurden mehrere Objecte gefunden, welche weder in der grossen Mondkarte von Schmidt, noch in jenen von Mädler und Lohrmann enthalten sind. Unter denselben sind namentlich zwei hervorzuheben, welche auch für kleinere Instrumente von nur 6 und 4 Zoll Oeffnung gut erkennbar erscheinen, und welche die Frage nahe legen, warum sie wohl von den genannten trefflichen Selenographen übersehen worden sein mögen, während diese viel kleinere Objecte mit aller Sorgfalt und Genauigkeit verzeichnet haben?

Das erste Object ist eine grosse Rille, welche die Wallebene Thebit nahe meridional durchzieht und eine Länge von etwa 28 Kilometern hat. Ich entdeckte dieselbe zu Ende März d. J. auf der Lick-Photographie von 27. Aug. I 888 (Mondalter $=20$ Tage). Es sei gestattet, diesbezüglich den Wortlaut meines Schreibens an Herrn Professor Holden vom 9. April d. J. anzufüren. »Anbei sende ich Ihnen eine schnell gefertigte, ziemlich treue Copie meiner Io fach vergrösserten Tuschirung von Thebit (südlich von Arzachel), welche ich in den letzten Tagen nach der schönen Lick-Aufnahme vom 27. August I 888 ausgeführt habe. Ich wählte, trotzdem ich noch inmitten anderer Arbeiten stehe, auch dieses Object, weil dasselbe im Inneren, von $\zeta$ gegen $\varepsilon$ (vergl. Neison) hin, eine Art Rille zeigt, die einem Bruch in der Sohle täuschend ähnlich sieht und weder bei Lohrmann oder Mädler, noch bei Schmidt irgendwie angedeutet ist. Diese Art Rille in Thebit, welche im nördlichen Theile gegen Osten hin zwei Abzweigungen zu besitzen scheint, stellt sich auf der genannten Photographie noch deutlicher als die von Triesnecker westlich liegende Rille dar und zeigt dem Ansehen nach einen völlig gleichen Character, In der Nacht vom 31. März zum I. April d. J, wo die Beleuchtungsverhältnisse für den Mond nahe dieselben wie am 27. August 1888 waren, konnte ich mich mit dem Steinheil'schen 6 Zöller trotz des niedrigen Mondstandes $\left(\delta=-25^{\circ}\right)$ und grosser Luftunruhe ziemlich sicher von der Realität jenes Bruches im Inneren von Thebit auch optisch überzeugen. Meine sofort mit Dr. H. J. Klein in Cöln eingeleitete Correspondenz ergab, dass auch dieser erfahrene Mondbeobachter eine solche Thebit-Rille nicht kennt*) und dass auch bei Gruithuisen nichts daruber $z u$ finden ist. Wir hätten also in diesem Falle eine photographisch entdeckte Rille, die jedoch nicht neu entstanden zu sein braucht, da man ihre bisherige Nichtwahrnehmung auch aus der, möglicherweise kurzen, Sichtbarkeitsdauer derselben und aus dem Umstande, dass die Astronomen gegen Morgen nicht gerne beobachten, erklären kann. Ich bemerke noch, dass Mädler und Neison den vom Krater $A$ nordwestlich liegenden kleinen Krater unrichtig an den Aussenwall von Thebit verlegt haben. Er liegt nach der Photographie am Innenwalle und so, dass er auch als zur Sohle gehörig (vergl. Schröter) betrachtet werden kann. Schmidt und Lohrmann zeichnen ihn ziemlich richtig. Dagegen muss der Höhenzug im Inneren von Thebit nach der Photographie entschieden anders als bei Schmidt aufgefasst werden «. - Herr Professor Holden antwortete am 29. April, dass er die Thebit-Rille auf dem Original-Negativ vom 27. August I888 verificirt habe und auf anderen Negativen Spuren von derselben erkenne.

Das zweite Object betrifft einen Mondkrater von etwa $4 \frac{1}{2}$ Kilometer Durchmesser, den ich am 22. Mai d. J. südlich von der Verbindungslinie Pallas-Triesnecker im Sinus Medii (südöstlich von Chladni in 0.06 westlicher Länge und 2.24 nördlicher Breite) auf der Lick-Photographie vom I 5. August I 888 (Mondalter $=8$ Tage) entdeckte und vergeblich auf den Karten von Schmidt, Mädler, Lohrmann und Neison suchte. Da ein Duplicat der bemerkten Platte in Prag nicht vorhanden ist und das dunkle, runde Object von nur $0.2 \mathrm{~mm}$ Durchmesser auf dem Negative auch ein Fehler desselben sein konnte, obwohl dies nach der Nuancirung des Flecks und seiner wallartigen Umgebung nicht wahrscheinlich erschien, so wurde wieder Herr Professor Holden am 23. Mai d. J. unter Beifügung einer 10 fach vergrösserten Zeichnung der betr. Partie um die Verificirung des gefundenen Objectes auf Grund der auf der Lick-

*) Auch der selır eifrige und erfolgreiche englische Mondheobachter, Herr Thos. Gwyn Elger in Bedford, welcher bald von mir verständigt wurde, konnte nichts auf eine Thebit-Rille Beziigliche, linden. 
Sternwarte vorhandenen Negative ersucht. Herr Director Holden antwortete am Io. Juni d. J. und constatirte, dass dieser Krater auch auf den Negativen vom 24. August, 2 2. September und 3 . November 1890 , welche einem Mondalter von $9^{\mathrm{d}} 1_{1^{\mathrm{h}}}^{\mathrm{h}}, 8^{\mathrm{d}} 4^{\mathrm{h}}$ und $2 \mathrm{I}^{\mathrm{d}} 5^{\mathrm{h}}$ entsprechen, sichtbar ist, also wirklich existirt. Ferner zeigte derselbe auf einer Postkarte vom 12. Juni an, dass er diesen Krater auch auf einem Silberdruck nach einem, an der Sternwarte in Mel. bourne aufgenommenen, Negative vom I. September 1873 (Mondalter $=9$ Tage) auffinden konnte. Der fragliche Krater war also schon im Jahre 1873 vorhanden und wurde trotz seiner Grösse i 8 Jahre lang von den eifrigsten Beobachtern übersehen. Es sei noch erwähnt, dass dieser Krater kurze Zeit nach dem $\mathbf{r}$. Viertel $z u$ suchen wäre und zwar, indem man die Verbindungslinie von Pallas zu Triesnecker halbirt und deren halbe Länge vom Halbirungspunkte aus senkrecht nach Süden hin aufträgt.

Ohne Zweifel versprechen die vorzüglichen Mondaufnahmen der Lick-Sternwarte bei gehöriger Ausnützung derselben noch überraschende Resultate für die Erkenntniss der Mondoberfiächen-Verhältnisse.

$$
\text { Prag } 189 \text { I Juli I. L. Weinek. }
$$

P. S. vom 3. Juli. Soeben ist von Herrn Professor Holden ein Brief, datirt vom I6. Juni d. J., an mich gelangt, in welchem derselbe mittheilt, dass er am I 5. Juni Nachts $10^{\text {h }}$ den neuen Krater auch optisch verificirt habe. Er er. wähnt aber nicht, ob dies mit dem 36- oder mit dem 12 Zöller geschah. Die betr. Stellen seines Schreibens lauten: »Your crater discovered on the $\mathrm{L}$. $\mathrm{O}$. negative is circular with an interior shadow, and situated in the centre of a raised mound (of its own lava ?) \& ... s So that your new crater is verified fully - and has a second crater near it."

Nach Herrn Professor Holden's beigegebener Skizze liegt der letztere nahe östlich vom photographisch entdeckten Krater und zwar am Rande des angeführten (weissen) Walles. $W$.

\section{Elemente und Ephemeride des Planeten (250) Bettina. \\ Von Carl Mönnichmeyer.}

Die in den letzten Jahrgängen des Berl. astr. Jahrbuchs veröffentlichten Elemente der Bettina, welche aus den Beobachtungen der beiden ersten Erscheinungen abgeleitet waren, stellten den Ort des Planeten in der dritten Erscheinung (1888) nur ungenügend dar, indem die Ephemeride eine Correction von $+28^{\mathrm{s}},-4^{\prime} 22^{\prime \prime}$ verlangte. Vor längerer Zeit berechnete ich nun die durch Jupiter bewirkten Störungen und versuchte nach der Methode der Bestimmung einer Bahn aus 3 vollständigen Beobachtungen „Gauss, Th. m. Art. 120 « aus den Normalörtern 1885 Sept. I 2 , 1886 Dec. 23 und I 888 März 6 Elemente abzuleiten. Der Versuch musste

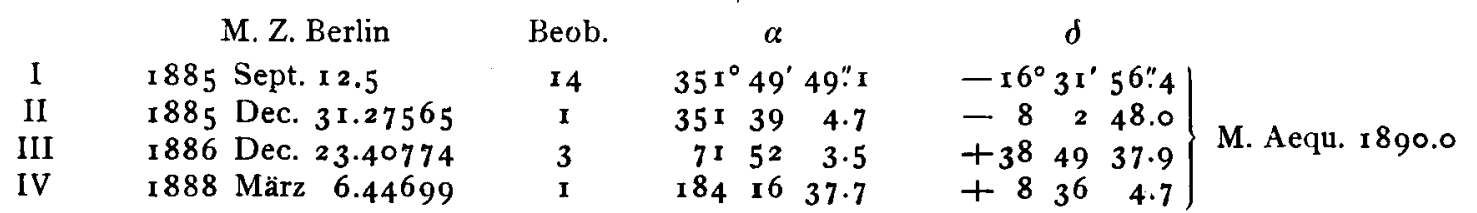

das Elementensystem A abgeleitet, welches bei 6 stelliger Rechnung im zweiten und vierten Ort die Fehler übrig liess (Normalort - Rechnung) :

$$
\text { II } \quad \partial \alpha=-3 " 1 \quad \partial \delta=-1.4 ; \quad \text { IV } \quad \partial \alpha=-3.9 \quad \partial \delta=-4.9 .
$$

Unter Zugrundelegung der alten Störungswerthe habe ich die durch Jupiter hervorgebrachten Störungen von 1887 Dec. 18 an neu berechnet und aus dem System A das System B erhalten:

A.

Epoche und Osculation 1885 Dec. 8.0 M. Z. Berlin.

$$
\begin{aligned}
& M=288^{\circ} \text { I I }^{\prime} 55^{\prime \prime} \mathrm{o} \\
& \omega=\begin{array}{lll}
62 & 8 & 3.4
\end{array} \\
& \delta=\begin{array}{lll}
26 & 9 & 3.4
\end{array} \text { M. Aequ. } 1890.0 \\
& i=12 \quad 5^{6} \quad 23.9 \\
& \varphi=72 \text { I } 56.4 \\
& \mu=634 " 34368 \\
& \log a=0.4984547
\end{aligned}
$$

als vergeblich aufgegeben werden, da nach io Hypothesen eine merkliche Annäherung nicht erreicht wurde. Ich glaube dies nicht unerwähnt lassen zu dürfen; sind die Zwischenzeiten sehr gross, und kommen die hypothetischen Annahmen von $y, u$ und $r$ den wirklichen Werthen zu wenig sprechender Mühe zum Ziel gelangen.

Mit Berücksichtigung der Störungen durch Jupiter wurde jetzt nach der Methode der Variation der Distanzen aus den Normalörtern : 